Keio J. Med. 23: 191-204, 1974

\title{
TOXICOLOGIC STUDIES OF ANTITUMOR AMINO ACID DERIVATIVES IN MICE AND RATS
}

\author{
KOJI FUKUSHIMA,* SOGEN ITOH** and SHIGESHI TOYOSHIMA* \\ * Division of Chemotherapy, Pharmaceutical Institute, School of Medicine, \\ Keio University, Shinanomachi, Shinjuku-ku, Tokyo 160, Japan \\ ** Department of Internal Medicine, The 2nd Tolyo National Hospital, \\ Higashigaoka, Meguro-ku, Tokyo, Japan
}

(Received for publication August 19, 1974)

\begin{abstract}
Toxicity of five antitumor amino acid derivatives, $N$ - $\beta$-naphthalenesulfonyl-DL-tryptophan(A-91), $\beta$-naphthylaminomethyl- $\gamma$-aminobutyric acid (A-144), N-ethylcarbaminomethyl-L-isoleucine (A-145), N-9-fluorenylacetyl-Lphenylalanine (A-192), and N-propionyl-L-valine (A-195) were studied in mice and rats. These amino acid derivatives (A-A derivatives), with the exception of A-192, were less toxic in mice and rats administered single intraperitoneally (ip) and orally (po).

In repeated-dose studies with the five A-A derivatives, the rats were treated ip with daily doses of one-third LD50 every day for 10 days. During the observation period, the rats treated with A-A derivatives, with the exception of A-195, showed no adverse signs and caused no body weight loss. However, some rats treated with A-195 developed poor apetite or anorexia, weight loss, and death during the two weeks observation period. Treatment of A-A derivatives caused no leukopenia, as in treatment of Mitomycin-C, and the other hematologic toxicity was slightly changed compared with the control groups.

Renal and hepatic functions were slightly affected in rats treated with A-A derivatives.

Severe lesions were noted in testes of rats treated with Mitomycin-C, but treatment with A-A derivatives caused moderate lesions in testes. All other tissues appeared to be normal.

These results indicate that the toxicity of A-A derivatives is less toxic than that of Mitomycin-C in animals.
\end{abstract}

\section{INTRODUCTION}

Five amino acid derivatives from about 350 compounds were shown to possess antitumor activity in vivo and in vitro from the primary screenings (Fukushima and Toyoshima). ${ }^{3}$ These compounds were also capable of inhibiting the growth 
of Ehrlich ascites carcinoma in mice. Further studies in antitumor activity of amino acid derivatives against various tumor systems indicate that some of these compounds were not only effective against Ehrlich carcinoma but ascites and solid Sarcoma 180, Nakahara-Fukuoka sarcoma, rat ascites hepatoma such AH 13, AH 7974, AH 60C and Yoshida sarcoma (Fukushima and Toyoshima). ${ }^{4}$ N-ethylcarbaminomethyl-L-isoleucine(A-145) was particularly active against various types tumors, except leukemia in vivo, and the inhibiting effect on RNA, DNA and protein in tumor cells.

On the basis of potent antitumor activity in mice and rats, five amino acid derivatives were selected for toxicological evaluation: $N-\beta$-naphthalene-sulfonylDL-tryptophan(A-91), $\beta$-naphthylaminomethyl- $\gamma$-aminobutyric acid(A-144), $\mathrm{N}$ ethylcarbaminomethyl-L-isoleucine (A-145), N-9-fluorenylacetyl-L-phenylalanine (A-192) and N-propionyl-L-valine (A-195). The chemical structures of five amino

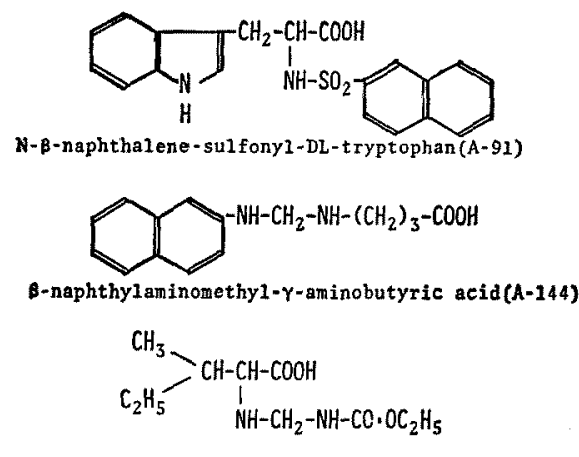

N-othylcarbaminonethyl-L-isoleucine $(A-145)$

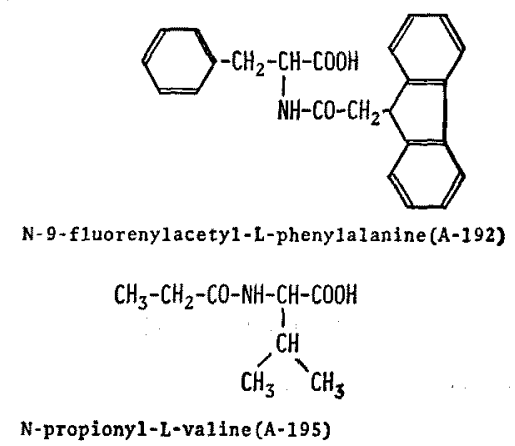

Fig. 1 Chemical structures of amino acid derivatives. 
acid derivatives (A-A derivatives) are shown in figure 1. Toxicologic data obtained in experiments performed with mice and rats are presented and the toxic effects of five A-A derivatives noted in the two species are compared with those of mitomycin-C, standard reference drug.

\section{MATERIALS AND METHODS}

Determination of LD50 in mice and rats :

Groups of 10 male ICR mice $(20-25 \mathrm{~g})$ and Donryu rats $(130-150 \mathrm{~g})$ were used in this study. Water was available ad libitum. All A-A derivatives suspended in $0.1 \%$ carboxymethylcellulose (CMC) and administered intraperitoneally(ip) and orally (po) for single-dose toxicity. Necropsies were conducted on mice and rats that died and on those which were killed after surviving the 14 day observation period. The method of Lichfield and Wilcoxon ${ }^{7}$ was used to caluculate the LD50 of A-145 ip and 95\% confidence limits.

Serial toxicity study of amino acid derivatives in mice and rats:

Doses of A-A derivatives were administered ip daily for 10 days to groups of 10 male mice and rats. The daily tested dose of each compound was one-third of LD50 given ip. All animals were examined daily for changes in physical condition and observed for 28-30 days. After treatment, daily food and water intake was estimated, during the first week, body weight was recorded twice weekly. During observation period, the animals were carefully observed for toxic signs and various parameters were studied for adverse effects. After the animals died or were killed, necropsies were performed and tissues were examined microscopically.

Routine hematologic tests included determination of hematocrit and hemoglobin values, total red blood cell count (rbc), white blood cell count(wbc), wbc differential, and thrombocyte count, and prothrombin time. Values for the following blood chemical constituents were also determined: blood glucose, blood urea nitrogen(BUN), serum creatinine, serum cholesterol, serum alkaline phosphatase, serum glutamic oxaloacetic transaminase(SGOT), serum glutamic pyrvic transaminase (SGPT), and serum protein, $\operatorname{sodium}\left(\mathrm{Na}^{+}\right), \operatorname{potassium}\left(\mathrm{K}^{+}\right)$and chloride $\left(\mathrm{Cl}^{-}\right)$. Tissue samples were fixed in $10 \%$ neutral buffered formaldehyde and embedded in paraffin. The tissues included liver, kidneys, pituitary gland, brain (three levels), heart, adrenal glands, thyroid glands, lung, spleen, testes, seminal vesicle, prostate gland, stomach, duodenum, pancreas, jejunum, thymus, ileum, urinary bladder, cecum, colon, straited muscle, skin, optic nerve, and bone 
marrow. Further necropsies were performed and tissues were examined microscopically.

\section{RESULTS}

Single-dose toxicity of amino acid derivatives:

Amino acid derivatives(A-A derivatives) were given ip to groups of 10 mice and 10 rats at dose levels of $100,150,200,500,750,1000,1250$, and $1500 \mathrm{mg} / \mathrm{kg}$. Orally administered A-A derivatives were given at dose levels of 1000, 1500, 2000, 3000,4000 , and $5000 \mathrm{mg} / \mathrm{kg}$. The vehicle for both ip and oral was $0.1 \% \mathrm{CMC}$. Because treatment with higher doses would have required the injection of a large volume of the test compound solution, determination of the median lethal dose of some A-A derivatives was not attempted. The LD50 of A-A derivatives in mice and rats treated by both routes are compared in table 1 . Death occurred from Day 2 through Day 4 after single ip administered doses and from Day 3 through Day 7 after single orally administered doses. Gross examination of these animals after death revealed hemorrhanges in one or more vital organs, the gastrointestinal system being slightly affected.

These results indicate that these A-A derivatives, with the exception of A-192, were less toxic in mice and rats when administered single ip and orally.

Repeated-dose studies with amino acid derivatives:

Repeated daily doses of A-91, A-144, A-145, A-192, A-195 and mitomycin-C

Table 1

Lethal dose (LD50) of selected amino acid derivatives in mice and rats

\begin{tabular}{clrr}
\hline \hline \multirow{2}{*}{$\begin{array}{c}\text { Compounds } \\
\text { No. }\end{array}$} & $\begin{array}{c}\text { Route of } \\
\text { Administration }\end{array}$ & \multicolumn{2}{c}{ LD50 (mg/kg) } \\
\cline { 3 - 4 } A-91 & Intraperitoneal & 850 & rats \\
& Oral & $>5000$ & 922 \\
A-144 & Intraperitoneal & 1050 & - \\
& Oral & $>5000$ & 710 \\
A-145 & Intraperitoneal & $1320(1168-1491)^{*}$ & - \\
& Oral & $>5000$ & - \\
A-192 & Intraperitoneal & 175 & 144 \\
& Oral & 1850 & - \\
A-195 & Intraperitoneal & 1500 & $>1500$ \\
& Oral & $>5000$ & - \\
& & &
\end{tabular}

* 95\% confidence limits 


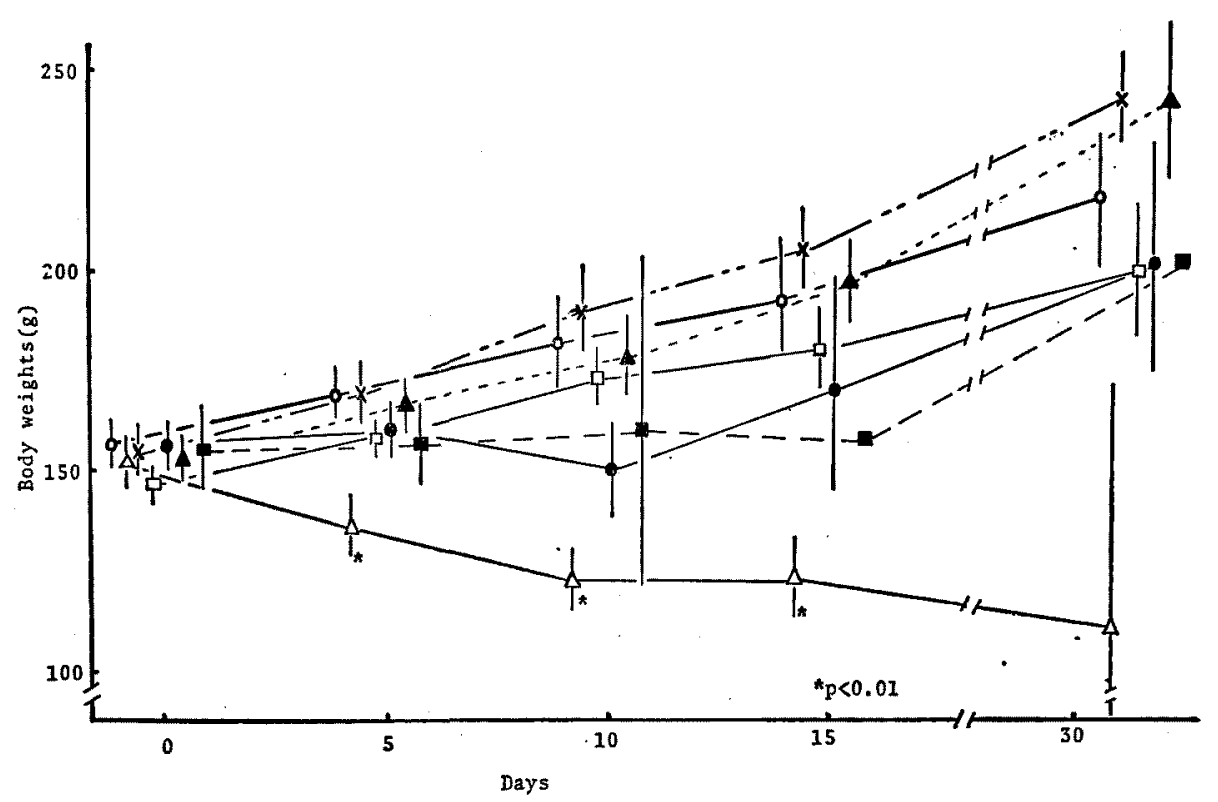

Fig. 2 Body weights of rats treated ip with 10 daily consecutive doses of amino acid derivatives. $O$, control; $X$, A-91 (307 $\mathrm{mg} / \mathrm{kg}) ; \square$, A-144 (236 $\mathrm{mg} / \mathrm{kg})$; $\bullet$, A-145 $(500 \mathrm{mg} / \mathrm{kg}) ; \Delta, A-192(48 \mathrm{mg} / \mathrm{kg}) ; \square, A-195(500 \mathrm{mg} / \mathrm{kg}) ; \triangle$, Mitomycin-C $(0.8 \mathrm{mg} / \mathrm{kg})$.

were given ip to groups of 10 male rats at doses of $307,236,500,48,500$, and $0.8 \mathrm{mg} / \mathrm{kg}$, respectively, for 10 consecutive days. Body weights are shown in figure 2. During the observation period, control rats and rats treated with A-A derivatives, with the exception of A-195, showed no adverse signs, maintained normal food consumption and did not show weight loss. However, loss of body weight of rats treated with mitomycin-C, was more than $10 \%$ of their initial body weight during observation periods. Several rats treated with A-195 developed poor appetites or anorexia, weight loss, and death during two weeks. Surviving rats recovered fully from all toxic effects and appeared healthy on Day 30.

The effects of A-A derivatives on the hematologic values of mice and rats are presented in figures $3,4,5$, and table 2 . The values of control animals appeared normal during the observation period. Slight fluctuation in various blood elements were noted. The erythrocyte count of groups after treatment with A-91 progressively increased Day 14 with a significant increase on Day 28 as compared to the control groups (fig. 3). The other A-A derivatives showed no sig- 
Table 2

Hematologic values (differential wbc) of rats treated ip with 10 daily

\begin{tabular}{|c|c|c|c|c|}
\hline$\underset{\text { tests }}{\text { Hematologic }}$ & Day & Control & $\begin{array}{c}\mathrm{A}-91 \\
(307 \mathrm{mg} / \mathrm{kg})\end{array}$ & $\begin{array}{c}\mathrm{A}-144 \\
(236 \mathrm{mg} / \mathrm{kg})\end{array}$ \\
\hline \multirow{5}{*}{ Staffeyte } & 0 & $6.7 \pm 6.0$ & $5.4 \pm 1.4$ & $7.5 \pm 1.1$ \\
\hline & 7 & $10.8 \pm 9.2$ & $10.1 \pm 0.9$ & $11.1 \pm 3.3$ \\
\hline & 13 & $4.8 \pm 2.0$ & $2.4 \pm 1.6$ & $3.0 \pm 0.7$ \\
\hline & 23 & $2.6 \pm 0.5$ & $2.5 \pm 1.8$ & $2.8 \pm 1.0$ \\
\hline & 28 & $3.2 \pm 0.8$ & $2.6 \pm 1.9$ & $2.0 \pm 0.4$ \\
\hline \multirow{5}{*}{ Segmentecyte } & 0 & $33.9 \pm 8.5$ & $28.6 \pm 7.0$ & $37.1 \pm 13.5$ \\
\hline & 7 & $35.8 \pm 6.9$ & $58.1 \pm 5.6^{* *}$ & $55.9 \pm 10.7^{*}$ \\
\hline & 13 & $22.1 \pm 4.9$ & $38.1 \pm 5.0^{* *}$ & $56.0 \pm 7.0^{* *}$ \\
\hline & 23 & $25.6 \pm 9.8$ & $40.8 \pm 7.6$ & $36.0 \pm 2.5$ \\
\hline & 28 & $27.4 \pm 7.6$ & $21.0 \pm 2.7$ & $32.0 \pm 11.1$ \\
\hline \multirow{5}{*}{ Lymphocyte } & 0 & $48.9 \pm 5.2$ & $55.5 \pm 5.6$ & $46.9 \pm 12.3$ \\
\hline & 7 & $39.8 \pm 4.7$ & $35.4 \pm 14.2$ & $25.7 \pm 10.9 *$ \\
\hline & 13 & $53.9 \pm 11.7$ & $42.1 \pm 3.6$ & $26.0 \pm 7.3^{* *}$ \\
\hline & 23 & $58.3 \pm 10.6$ & $43.1 \pm 7.4$ & $44.3 \pm 4.8$ \\
\hline & 28 & $47.0 \pm 15.9$ & $72.4 \pm 5.4^{*}$ & $38.2 \pm 16.1$ \\
\hline \multirow{5}{*}{ Monocyte } & 0 & $8.0 \pm 2.2$ & $8.8 \pm 3.7$ & $7.3 \pm 2.5$ \\
\hline & 7 & $11.3 \pm 3.1$ & $4.9 \pm 0.6^{* *}$ & $6.1 \pm 2.5^{*}$ \\
\hline & 13 & $14.1 \pm 7.1$ & $13.6 \pm 2.5$ & $11.3 \pm 1.5$ \\
\hline & 23 & $11.5 \pm 1.5$ & $9.1 \pm 1.5$ & $11.7 \pm 2.5$ \\
\hline & 28 & $8.4 \pm 3.4$ & $2.3 \pm 1.0^{* *}$ & $8.5 \pm 3.5$ \\
\hline \multirow{5}{*}{ Eosinophil } & 0 & $2.8 \pm 3.6$ & $1.8 \pm 0.1$ & $1.2 \pm 0.2$ \\
\hline & 7 & $1.8 \pm 1.1$ & $0.8 \pm 0.5$ & $0.8 \pm 0.2$ \\
\hline & 13 & $3.1 \pm 1.3$ & $1.9 \pm 0.5$ & $2.2 \pm 0.8$ \\
\hline & 23 & $2.0 \pm 0.7$ & $2.9 \pm 1.5$ & $2.2 \pm 1.0$ \\
\hline & 28 & $1.5 \pm 0.7$ & $1.5 \pm 0.5$ & $1.7 \pm 1.3$ \\
\hline \multirow{5}{*}{ Basophil } & 0 & $0.5 \pm 0$ & 0.8 & 1.3 \\
\hline & 7 & $0.8 \pm 0.2$ & - & $0.6 \pm 0.2$ \\
\hline & 13 & $1.3 \pm 0.8$ & $1.9 \pm 1.0$ & $1.5 \pm 1.1$ \\
\hline & 23 & $1.3 \pm 0.3$ & $1.6 \pm 0.2$ & $1.3 \pm 0.8$ \\
\hline & 28 & $2.0 \pm 0.8$ & 1.0 & $0.7 \pm 0.2$ \\
\hline
\end{tabular}

These values represent differential wbe $(\%) . * \mathrm{P}<0.05, * * \mathrm{P}<0.01$. 
consecutive doses of selected amino acid derivatives

\begin{tabular}{|c|c|c|c|}
\hline $\begin{array}{c}\mathrm{A}-145 \\
(500 \mathrm{mg} / \mathrm{kg})\end{array}$ & $\begin{array}{c}\mathrm{A}-192 \\
(48 \mathrm{mg} / \mathrm{kg})\end{array}$ & $\begin{array}{c}\mathrm{A}-195 \\
(500 \mathrm{mg} / \mathrm{kg})\end{array}$ & $\begin{array}{l}\text { Mitomyein-C } \\
(0.8 \mathrm{mg} / \mathrm{kg})\end{array}$ \\
\hline $5.7 \pm 1.6$ & $3.8 \pm 2.0$ & $8.6 \pm 4.1$ & $5.3 \pm 0.9$ \\
\hline $5.8 \pm 1.4$ & $6.5 \pm 2.1$ & $13.6 \pm 11.4$ & $4.8 \pm 2.6$ \\
\hline 2.3 & $3.1 \pm 1.6$ & $9.7 \pm 5.9$ & 3.0 \\
\hline 3.5 & $1.4 \pm 1.0$ & $8.7 \pm 8.8$ & - \\
\hline 2.5 & $3.6 \pm 0.6$ & $10.0 \pm 7.5$ & - \\
\hline $25.7 \pm 1.6$ & $33.9 \pm 13.3$ & $28.9 \pm 8.6$ & $32.7 \pm 8.7$ \\
\hline $44.9 \pm 3.6$ & $52.3 \pm 14.0$ & $43.4 \pm 10.6$ & $51.8 \pm 11.2^{*}$ \\
\hline 32.5 & $40.4 \pm 7.0$ & $31.3 \pm 12.5$ & 63.5 \\
\hline 24.5 & $20.9 \pm 7.2$ & $28.0 \pm 20.2$ & - \\
\hline 15.5 & $15.9 \pm 6.2$ & $18.5 \pm 15.4$ & - \\
\hline $59.0 \pm 9.6$ & $40.7 \pm 13.4$ & $55.2 \pm 8.5$ & $51.4 \pm 9.7$ \\
\hline $40.0 \pm 4.6$ & $31.8 \pm 15.4$ & $31.0 \pm 6.8$ & $37.7 \pm 10.8$ \\
\hline 51.8 & $45.9 \pm 7.8$ & $44.8 \pm 3.7$ & 26.5 \\
\hline 55.3 & $66.1 \pm 8.5$ & $49.3 \pm 16.6$ & - \\
\hline 74.0 & $75.9 \pm 5.2^{*}$ & $49.7 \pm 12.7$ & - \\
\hline $7.5 \pm 3.0$ & $10.6 \pm 3.4$ & $5.6 \pm 1.7$ & $6.6 \pm 1.4$ \\
\hline $8.0 \pm 2.7$ & $6.2 \pm 2.4^{*}$ & $7.6 \pm 4.6$ & $5.4 \pm 2.3^{*}$ \\
\hline 12.5 & $8.8 \pm 3.1$ & $11.5 \pm 3.2$ & 7.0 \\
\hline 6.8 & $8.5 \pm 0.7^{*}$ & $11.1 \pm 3.0$ & - \\
\hline 6.5 & $3.4 \pm 0.4^{*}$ & $3.3 \pm 1.9$ & - \\
\hline $2.1 \pm 0.8$ & $0.7 \pm 0.4$ & $1.7 \pm 1.3$ & $2.0 \pm 1.4$ \\
\hline $1.3 \pm 0.5$ & $1.7 \pm 1.3$ & 0.5 & 0.5 \\
\hline 0.8 & $1.8 \pm 0.6$ & $1.3 \pm 0.8$ & - \\
\hline 0.8 & $1.3 \pm 0.6$ & $3.3 \pm 2.3$ & - \\
\hline 1.0 & $1.5 \pm 1.1$ & $1.0 \pm 0.8$ & - \\
\hline 0.5 & 0.8 & $0.6 \pm 0.2$ & $0.7 \pm 0.2$ \\
\hline 1.8 & 0.5 & 1.0 & 1.0 \\
\hline 2.5 & 1.0 & $1.3 \pm 0.6$ & 1.5 \\
\hline 0.5 & 2.0 & $0.7 \pm 0.2$ & 2.0 \\
\hline 1.0 & 0.5 & 0.8 & - \\
\hline
\end{tabular}




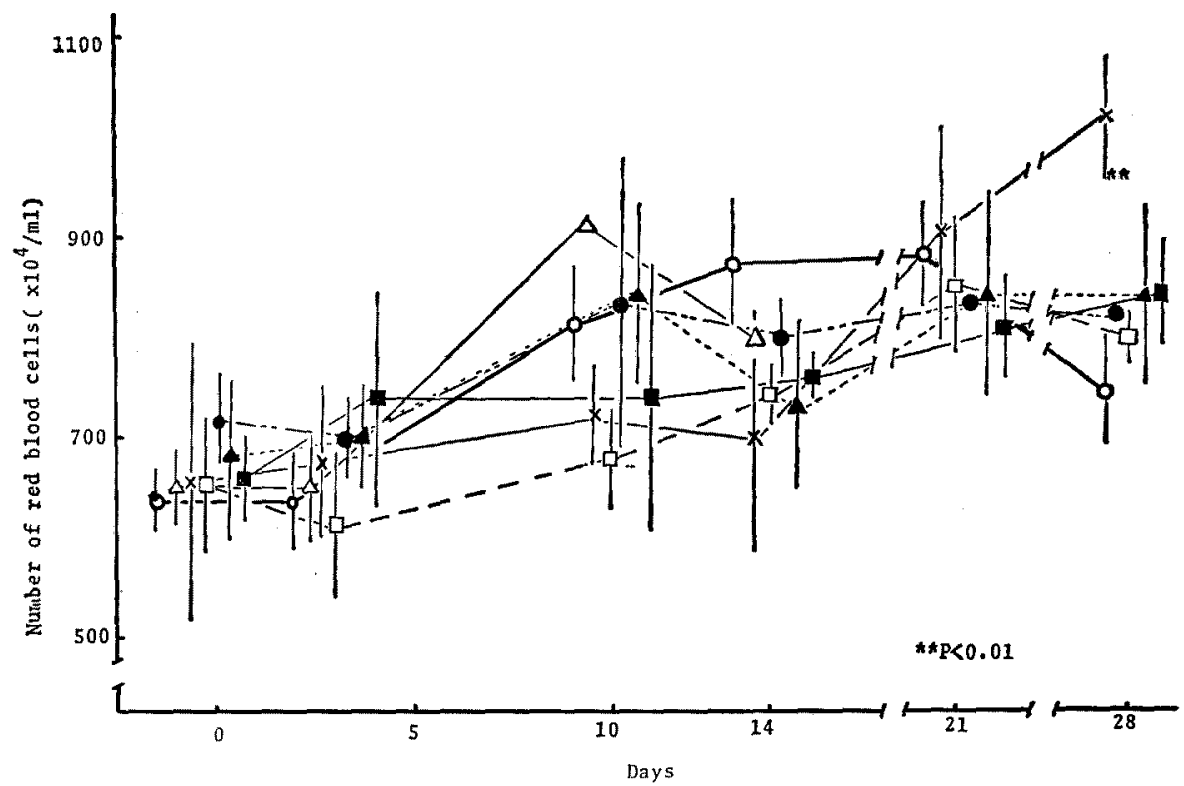

Fig. 3 Effects of selected amino acid derivatives on the hematologic values (red blood cells) of rats treated ip with 10 daily consecutive doses. $O$, control; $\times, A-91$ $(307 \mathrm{mg} / \mathrm{kg}) ; \square$, A-144 $(236 \mathrm{mg} / \mathrm{kg}) ; \bullet, A-145(500 \mathrm{mg} / \mathrm{kg}) ; \Delta, A-192$ $(48 \mathrm{mg} / \mathrm{kg}) ; \mathrm{E}, \mathrm{A}-195(500 \mathrm{mg} / \mathrm{kg}) ; \triangle$, Mitomycin-C $(0.8 \mathrm{mg} / \mathrm{kg})$.

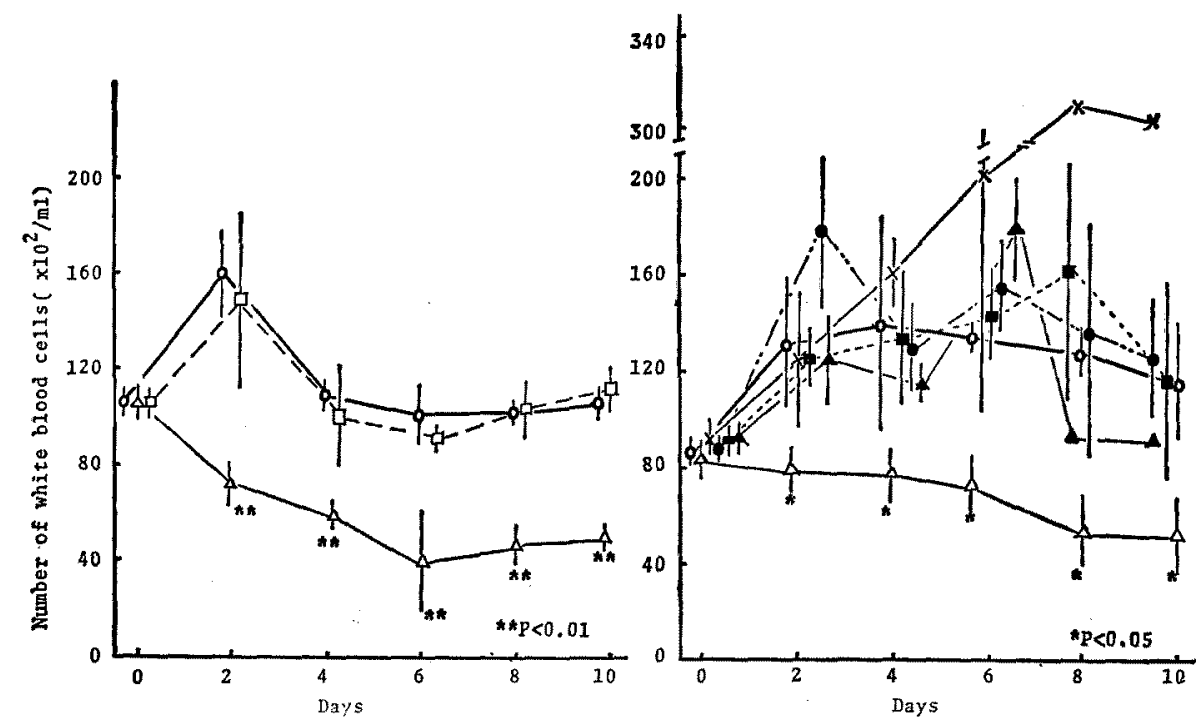

Fig. 4 Effects of selected amino acid derivatives on the hematologic values (white blood cells) of mice treated ip with 10 daily consecutive doses. $O$, control; $\times$, A-91 $(283 \mathrm{mg} / \mathrm{kg}) ; \square$, A-144 (350 $\mathrm{mg} / \mathrm{kg}) ; \square$, A-145 $(500 \mathrm{mg} / \mathrm{kg})$; A, A-195 $(500 \mathrm{mg} / \mathrm{kg}) ; \triangle$, Mitomycin-C $(2.5 \mathrm{mg} / \mathrm{kg}) ; \bullet, A-192(58 \mathrm{mg} / \mathrm{kg})$. 


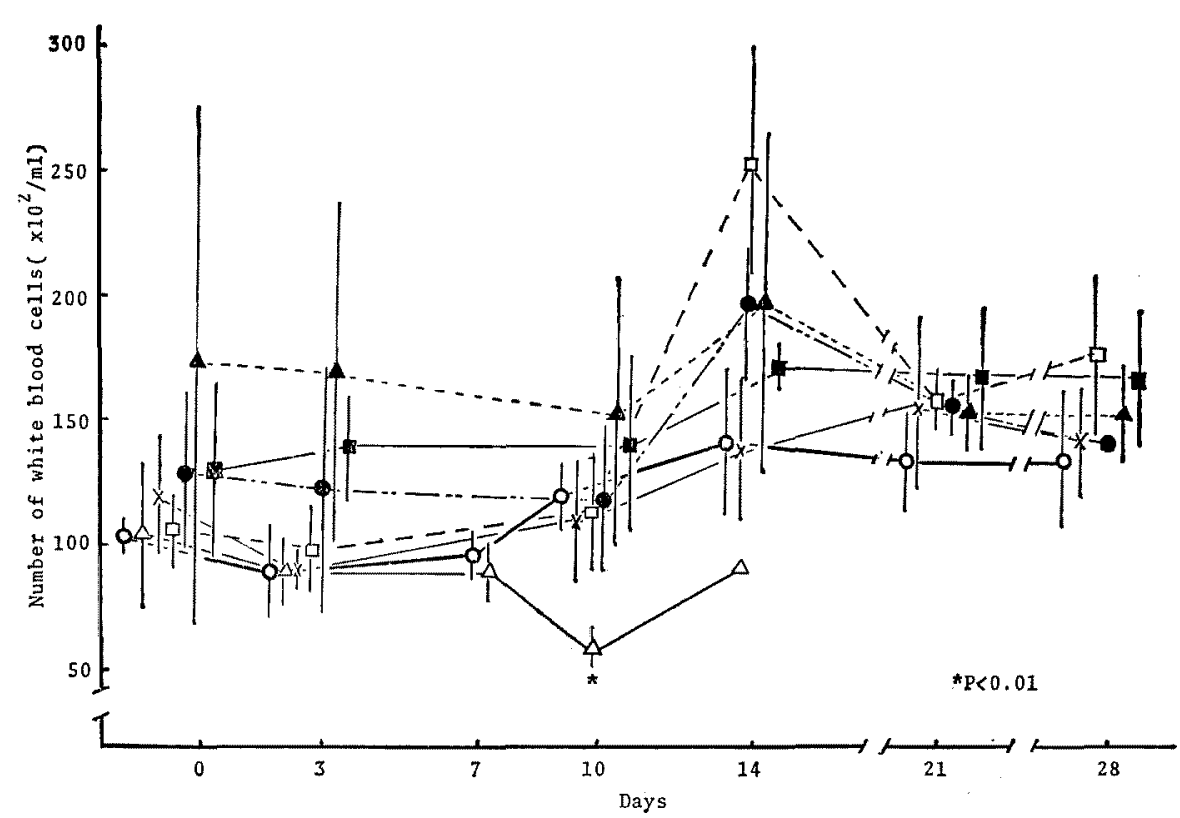

Fig. 5 Effect of selected amino acid derivatives on the hematologic values (white blood cells) of rats treated ip with daily 10 consecutive doses. $O$, control; $\times$, A-91 (307 mg/kg); $\square$, A-144 (236 mg/kg); A, A-145 (500 mg/kg); $\Delta$, A-192 (48 mg/kg); $\mathbf{D}$, A-195 $(500 \mathrm{mg} / \mathrm{kg}) ; \triangle$, Mitomycin-C $(0.8 \mathrm{mg} / \mathrm{kg})$.

nificant changes in the erythrocyte count.

The number of leukocytes in mice and rats treated with A-A derivatives are shown in fig. 4 and fig. 5. Depletion of leukocyte in aminals caused by treatment with A-A derivatives was not observed during observation periods. Leukocyte count showed a progressive increase in mice treated with A-91 after Day 4. These changes were not clearly defined, but it may be assumed that mice become infected with microbes during tests. Mitomycin-C treatments caused leukopenia within 10 days, in all mice and rats. The leukopenia was more severe in mice treated with mitomycin-C and persisted during treatments. Since the rats died before blood samples were taken and the leukocyte counts were not determined, it is uncertain whether the leukopenia was associated with a decrease in the total number of lymphocytes after Day 13 (table 2). Observations on Day 28 showed a large increase in the number of lymphocytes in A-91, A-145, and A-192. Tests on Day 7 and 13 show that A-144 caused a decrease in lymphocytes similar to that of cases treated with mitomycin-C. The number of peripheral segmentcytes also increased in rats treated with A-91, A-144 and A-192 similar to those caused by mitomycin-C. 
Table $s$

Hematologic values (hemoglobin, hematocrit, thrombocyte, prothrombin time) of rats treated ip with 10 daily consecutive doses of selected amino acid derivatives

\begin{tabular}{cccccc}
\hline \hline $\begin{array}{c}\text { Compounds } \\
\text { No. }\end{array}$ & $\begin{array}{c}\text { Dose } \\
(\mathrm{mg} / \mathrm{kg}, \mathrm{ip})\end{array}$ & $\begin{array}{c}\text { Hemoglobin } \\
(\mathrm{g} / \mathrm{dl})\end{array}$ & $\begin{array}{c}\text { Hematocrit } \\
(\%)\end{array}$ & $\begin{array}{c}\text { Thrombocyte } \\
\left(\times 10^{4} / \mathrm{mm}^{3}\right)\end{array}$ & $\begin{array}{c}\text { Prothrombin } \\
\text { time }(\mathrm{sec})\end{array}$ \\
\hline Control & & $14.7 \pm 0.7$ & $41.7 \pm 1.2$ & $25.9 \pm 2.2$ & $12.9 \pm 0.8$ \\
A-91 & 307 & $14.7 \pm 0.8$ & $40.0 \pm 1.9$ & $33.5 \pm 7.5$ & $12.8 \pm 1.9$ \\
A-144 & 236 & $13.9 \pm 1.1$ & $40.4 \pm 1.9$ & $36.8 \pm 5.1^{* *}$ & $12.7 \pm 1.5$ \\
A-145 & 500 & $13.2 \pm 1.5$ & $37.0 \pm 2.5$ & $22.8 \pm 3.5$ & $11.4 \pm 1.4$ \\
A-192 & 48 & $14.9 \pm 0.7$ & $42.4 \pm 1.6$ & $34.0 \pm 6.9^{*}$ & $13.5 \pm 1.1$ \\
\hline
\end{tabular}

These values represents on Day 30 .

Significant difference from the controls $\left(* \mathrm{P}<0.05,{ }^{* *} \mathrm{P}<0.01\right)$.

Table 4

Effects of selected amino acid derivatives on the blood chemical values

\begin{tabular}{cccccc}
\hline $\begin{array}{c}\text { Compounds } \\
\text { No. }\end{array}$ & $\begin{array}{c}\text { Dose } \\
(\mathrm{mg} / \mathrm{kg}, \mathrm{ip})\end{array}$ & $\begin{array}{c}\text { Protein } \\
\mathrm{A} / \mathrm{G}\end{array}$ & $\begin{array}{c}\text { BUN } \\
(\mathrm{mg} / \mathrm{dl})\end{array}$ & $\begin{array}{c}\text { Creatinine } \\
(\mathrm{mg} / \mathrm{dl})\end{array}$ & $\begin{array}{c}\text { Cholesterol } \\
(\mathrm{mg} / \mathrm{dl})\end{array}$ \\
\hline Control & & $1.07 \pm 0.33$ & $26.2 \pm 2.2$ & $0.70 \pm 0.04$ & $63.5 \pm 9.9$ \\
A-91 & 307 & $1.27 \pm 0.15$ & $21.5 \pm 2.6^{* * \mathrm{c})}$ & $0.73 \pm 0.10$ & $44.7 \pm 9.1^{* *}$ \\
A-144 & 236 & $0.92 \pm 0.14$ & $21.5 \pm 1.2^{* *}$ & $0.69 \pm 0.05$ & $65.3 \pm 6.9$ \\
A-145 & 500 & $0.82 \pm 0.19$ & $23.1 \pm 1.6^{*}$ & $0.67 \pm 0.06$ & $52.3 \pm 3.1$ \\
A-192 & 48 & $0.87 \pm 0.17$ & $24.1 \pm 1.4$ & $0.65 \pm 0.04$ & $51.5 \pm 4.6^{*}$ \\
A-195 & 500 & 0.71 & 21.3 & 0.70 & 54.0 \\
Mitomycin-C & 0.8 & 0.44 & 31.0 & 0.78 & 136.0 \\
\hline
\end{tabular}

a) Karmen units. b) King-Armstrong units. c) Significant difference from These parameters were determined on Day 30.

Table 5

Relative organ weights of rats treated ip with 10 daily consecutive doses of selected amino acid derivatives

\begin{tabular}{cccccccc}
\hline \hline $\begin{array}{c}\text { Compounds } \\
\text { No. }\end{array}$ & $\begin{array}{c}\text { Dose } \\
(\mathrm{mg} / \mathrm{kg}, \\
\mathrm{ip})\end{array}$ & $\begin{array}{c}\text { Brain } \\
(\mathrm{mg})\end{array}$ & $\begin{array}{c}\text { Hypo- } \\
\text { physis } \\
(\mathrm{mg})\end{array}$ & $\begin{array}{c}\text { Thyroid } \\
(\mathrm{mg})\end{array}$ & $\begin{array}{c}\text { Thymus } \\
(\mathrm{mg})\end{array}$ & $\begin{array}{c}\text { Heart } \\
(\mathrm{mg})\end{array}$ & $\begin{array}{c}\text { Lung } \\
(\mathrm{g})\end{array}$ \\
\hline Control & & $736.5 \pm 39.3$ & $2.9 \pm 0.5$ & $9.5 \pm 1.9$ & $164.2 \pm 48.0$ & $399.5 \pm 50.9$ & $0.67 \pm 0.21$ \\
A-91 & 307 & $668.0 \pm 60.5^{* *}$ & $2.8 \pm 0.7$ & $7.3 \pm 1.3$ & $176.7 \pm 33.0$ & $432.3 \pm 45.8$ & $0.69 \pm 0.11$ \\
A-144 & 236 & $779.6 \pm 45.8^{*}$ & $2.8 \pm 0.6$ & $8.0 \pm 2.2$ & $187.2 \pm 59.0$ & $405.5 \pm 41.5$ & $1.08 \pm 0.50$ \\
A-145 & 500 & $782.8 \pm 26.9$ & $4.1 \pm 0.7^{*}$ & $7.6 \pm 2.0$ & $165.2 \pm 13.7$ & $424.7 \pm 39.2$ & $0.72 \pm 0.11$ \\
A-192 & 48 & $641.3 \pm 44.2^{* *}$ & $2.4 \pm 0.7$ & $7.9 \pm 1.5$ & $188.3 \pm 34.9$ & $403.5 \pm 50.1$ & $0.77 \pm 0.29$ \\
A-195 & 500 & 788.2 & 5.4 & 8.4 & 114.3 & 419.7 & 0.69 \\
Mitomycin-C & 0.8 & $1345.8 \pm 55.4^{* *}$ & 2.7 & $12.1 \pm 1.8$ & $133.4 \pm 36.9$ & $471.2 \pm 65.4$ & $1.91 \pm 0.17 * *$ \\
\hline
\end{tabular}

These values represent weights per $100 \mathrm{~g}$ of body weight. 
Treatment of A-A derivatives did not cause any changes in the hematocrit and hemoglobin values, however, the thrombocyte count increased slightly in rats treated with A-144 and A-192 Day 30 observations, as shown in table 3.

The effects of A-A derivatives on the blood chemical values and serum electrolyte levels in rats are summarized in table. 4. A-91 caused decreases in BUN and cholesterol values. A-144 caused decreases in BUN, alkaline phosphatase, and glucose level. Decreases in alkaline phosphatase were not noted on Day 14. A-145 caused decreases in BUN and SGPT values, but the other experiments showed that did not cause any change in SGPT values as compared with untreated groups. Therefore, these changes in SGPT may be considered slight. A-192 caused decreases in cholesterol and glucose level. Since rats treated with A-195 and mitomycin-C died before these parameters were determined, these effects are uncer-

of rats treated ip with 10 daily consecutive doses

\begin{tabular}{|c|c|c|c|c|c|c|}
\hline \multirow{2}{*}{$\begin{array}{l}\text { Glucose } \\
\text { (mg/dl) }\end{array}$} & \multicolumn{2}{|c|}{ Transminase } & \multirow{2}{*}{$\begin{array}{l}\text { Alkaline } \\
\text { phosphataseb) }\end{array}$} & \multicolumn{3}{|c|}{ Serum electrolytes $(\mathrm{mEq} / \mathrm{L})$} \\
\hline & SGOT & SGPT & & $\mathrm{Na}^{+}$ & $\mathrm{K}^{+}$ & $\mathrm{Cl}^{-}$ \\
\hline $135.7 \pm 13.3$ & $109.7 \pm 5.1$ & $25.0 \pm 3.1$ & $35.9 \pm 14.1$ & $142.9 \pm 1.3$ & $5.6 \pm 1.0$ & $101.4 \pm 3.1$ \\
\hline $123.4 \pm 7.4$ & $105.9 \pm 9.4$ & $24.2 \pm 5.0$ & $32.1 \pm 17.2$ & $143.7 \pm 1.8$ & $5.4 \pm 0.3$ & $103.6 \pm 1.9$ \\
\hline $113.3 \pm 5.6^{* *}$ & $103.7 \pm 14.1$ & $22.2 \pm 4.1$ & $16.1 \pm 3.8^{* *}$ & $143.2 \pm 1.2$ & $5.7 \pm 0.8$ & $103.5 \pm 1.6$ \\
\hline $119.5 \pm 17.2$ & $102.7 \pm 7.4$ & $16.3 \pm 0.9^{* *}$ & $33.6 \pm 24.0$ & $144.0 \pm 1.0$ & $6.2 \pm 0.5$ & $103.2 \pm 1.0$ \\
\hline $115.0 \pm 6.0^{*}$ & $102: 3 \pm 5.7$ & $26.0 \pm 6.8$ & $25.8 \pm 6.1$ & $144.3 \pm 1.4$ & $5.3 \pm 0.5$ & $102.5 \pm 1.3$ \\
\hline $121.7 \pm 12.1$ & 94.0 & 25.0 & 20.1 & - & 4.8 & 99.0 \\
\hline 86.3 & 123.0 & 32.0 & 40.2 & 141.0 & 6.6 & 100.0 \\
\hline
\end{tabular}

the controls $(* \mathrm{P}<0.05, * * \mathrm{P}<0.01)$.

\begin{tabular}{ccccccc}
\hline \hline $\begin{array}{c}\text { Liver } \\
(\mathrm{g})\end{array}$ & $\begin{array}{c}\text { Spleen } \\
(\mathrm{mg})\end{array}$ & $\begin{array}{c}\text { Adrenal } \\
(\mathrm{mg})\end{array}$ & $\begin{array}{c}\text { Kidney } \\
(\mathrm{g})\end{array}$ & $\begin{array}{c}\text { Testis } \\
(\mathrm{g})\end{array}$ & $\begin{array}{c}\text { Vesicula } \\
\text { seminalis } \\
(\mathrm{mg})\end{array}$ & $\begin{array}{c}\text { Prostate } \\
(\mathrm{mg})\end{array}$ \\
\hline $4.35 \pm 0.71$ & $247.0 \pm 50.9$ & $16.9 \pm 2.5$ & $0.85 \pm 0.11$ & $0.93 \pm 0.16$ & $356.1 \pm 118.2$ & $132.6 \pm 37.7$ \\
$4.32 \pm 0.46$ & $246.5 \pm 30.7$ & $15.3 \pm 3.2$ & $0.83 \pm 0.08$ & $0.90 \pm 0.14$ & $393.7 \pm 143.4$ & $127.3 \pm 23.5$ \\
$4.55 \pm 0.39$ & $251.7 \pm 34.2$ & $19.9 \pm 4.6$ & $0.85 \pm 0.07$ & $0.95 \pm 0.18$ & $325.7 \pm 132.8$ & $106.4 \pm 47.6$ \\
$4.85 \pm 0.61$ & $245.8 \pm 7.9$ & $21.1 \pm 4.7$ & $0.87 \pm 0.13$ & $0.91 \pm 0.15$ & $257.6 \pm 137.9$ & $96.8 \pm 58.3$ \\
$4.11 \pm 1.03$ & $279.1 \pm 40.2$ & $16.9 \pm 3.3$ & $0.83 \pm 0.13$ & $0.75 \pm 0.18^{*}$ & $346.2 \pm 102.4$ & $114.1 \pm 39.6$ \\
5.37 & 358.1 & 30.0 & 0.94 & 1.11 & 219.2 & 101.5 \\
$5.78 \pm 1.59^{*}$ & $183.7 \pm 17.9 *$ & $29.6 \pm 4.0^{* *}$ & $1.32 \pm 0.39^{* *}$ & $0.51 \pm 0.13^{* *}$ & $87.0 \pm 35.1^{* *}$ & 59.9 \\
\hline
\end{tabular}

Significant difference from the controls $(* \mathrm{P}<0.05, * * \mathrm{P}<0.01)$. 
tain. Low dose toxicological studies of A-195 are now under investigation.

These results suggest that hepatic and renal functions were slightly affected in rats treated with $\mathrm{A}-\mathrm{A}$ derivatives.

The relative organ weights of control rats and rats treated with A-A derivatives are summarized in table 5 . The relative weight of liver, kidney, lung, and adrenal glands of rats treated with mitomycin-C appeared to be higher than those of rats untreated or treated A-A derivatives. On the other hand, the relative weight of spleen and testes in rats with mitomycin-C appeared to be lower than those of the controls. The relative weights of other organs were not significantly altered. The pathologic changes occuring in the tissue of the control rats and rats treated with A-A derivatives were examined. The control rats were relatively free of any lesions except slight inflammatory lesions in lungs. Microscopic lesions in tissues of rats treated with mitomycin-C were numerous. ${ }^{9}$ The mucosa of the glandular stomach and colon were severely affected. In sternal bone marrow, the nucleated hepatopoielic cells were reduced in quantity and part of the bone marrow was degenerative. Though severe lesions were noted in testes of rats treated with mitomycin-C, treatment with $\mathrm{A}-\mathrm{A}$ derivatives caused only moderate lesions in testes. All other tissues appeared to be normal.

\section{DISCUSSION}

Amino acid derivatives (A-A derivatives), with the exception of A-192, were far less toxic in mice and rats when administered single ip or po. There was great difference between the ip and oral LD50s. Toxcity (based on the median lethal dose) in mice by single ip injection decreased in the following order: A-192 $>A-91>A-144>A-145>A-195$ (table 1). It would seem that high toxicity of A-192 can be attributed to be the fluorenylacetyl moiety, for phenylalanine is less toxic. However, acute toxicity of A-192 was less toxic than that of methotrexate, ${ }^{2}$ pteroylglutamic acid analog with antitumor activity, in mice and rats. From the studies of single dose in mice and rats it was clear that the toxicity of these A-A derivatives is delayed, for no deaths occurred on the day of administration but occurred on the second day or later. Several rats treated with A-195 developed poor apetites or anorexia, weight loss, and death during two weeks. On the other hand, treatments with mitomycin-C caused a protracted intoxiation that is delayed in onset and characterized by anorexia, steady loss in weight diarrhea, dehydration, and delayed death. Lesions in testes were the common pathologic changes in rats treated with A-A derivatives but lesions in testes were not as severe as in rats treated with mitomycin-C. 
Philips et al $^{9}$ have reported that lesions in hematopoietic tissues and the intestinal epithelium are the pathologic changes in rats, dogs, and monkeys treated with mitomycin-C. They probably account for most fatalities in these species as well as the major signs of toxicity in patients (Colsky et al $^{1}$ ). Philips et $a^{10}$ also have reported that the high susceptibility of the intestinal epithelium of experimental animals to damage by the quinazoline antifolate is similar to the sensitivity of the same tissue methotrexate ${ }^{8}$ and aminopterin.11

Rapidly proliferating tissue-bone marrow, lymphoid tissue, and intestinal epithelium- were not necessarily sensitive to the cytotoxicity of A-A derivatives. These findings clearly indicate that A-A derivatives do not cause thrombocytepenia, leukopenia, and anemia in animals. Mitomycin-C treatments caused leukopenia within 10 days in mice and rats, accompanied by decreases in the total numbers of the neurophils and the lymphocytes.

Treatment with A-A derivatives did not cause any changes in the hematocrit and hemoglobin values, however, the thrombocyte count increases slightly in rats treated with A-144 and A-192 on Day 30 observations (Table 3).

Toxic effects on renal and hepatic functions were slightly affected in rats treated with A-A derivatives; treatment with A-A derivatives did not caused increases in alkaline phosphatase, SGPT, BUN and serum creatinine noted by such antitumor agents as cyclophosphamide ${ }^{6}$ or actinomycin D. ${ }^{5}$

These results obtained in the present study suggest that toxicity of amino acid derivatives (A-A derivatives) is less than mitomycin-C in animals.

\section{REFERENCES}

1. Colosky, C. J., Escher, G. C. and Evancs, A.: Preliminary clinical pharmacology of mitomycin C. Proc. Am. Assoc. Cancer Res. 3: 13, 1959

2. Ferguson, F. C., Thiersch, J. B. and Philips, F. S.: The action of 4-amino-N10methyl-pteroylglutamic acid in mice, rats, and dogs. J. Pharmacol, Exp. Ther. 98: 293-299, 1950

3. Fukushima, K., Seto, Y., Fujita, H., Nakamura, Y., Toyoshima, S. and Kanao, S.: Antitumor effect of new amino acid analogues. Int. Congr. Chemother. Proc. 7th. Prague. 1971, pp 103-105

4. Fukushima, K. and Toyoshima, S.: Antitumor activity of selected amino acid derivatives against various tumor systems. Cancer Chemother. Rep. in press.

5. Marlow, M. and Henry, M. C.: Preclinical toxicologic study of actinomycin D. Cancer Chemother. Rep. 4: 77-84, 1973

6. Lee, C. C., Castles, T. R. and Kintner, L. D.: Single-dose toxicity of cyclophosphamide in dogs and monkeys. Cancer Chemother. Rep. 4: 51-76, 1973

7. Litchfield, J. T. and Wilcoxon, F.: A simplified method of evaluating dose-effect experiments. J. Pharmacol. Exp. Therap. 96: 99-113, 1949

8. Philips, F. S., Thiersch, J. B. and Ferguson, F. C.: Studies of the action of 4amino-pteroylglutamic acid and its congeners in mammals. Ann. NY Acad. Sci. 52: $1349-1359,1950$ 
9. Philips, F. S., Schwartz, H. S. and Sternberg, S. S.: Pharmacology of mitomycin C. I. Toxicity and pathologic effects. Cancer Res. 20:1354-1369, 1960

10. Philips, F. S., Sternberg, S. S., Sodergren, J. E. and Vidal, P.: Toxicologic studies of the 2,4-diamino-quinazoline antifolate, methasquin(NSC-122870). Cancer Chemother. Rep. 55: 35-42, 1971

11. Werkheiser, W. C.: The relation of folic acid reductase to aminopterin toxicity. J. Pharmacol. Exp. Ther. 137: 167-172, 1962 